8th Alexander Friedmann International Seminar

on Gravitation and Cosmology

International Journal of Modern Physics: Conference Series

Vol. 3 (2011) 281-285

(C) World Scientific Publishing Company

DOI: $10.1142 /$ S2010194511001371

\title{
CONFIRMATION OF COSMOLOGICAL BOUNCES PREDICTED BY ALEXANDER FRIEDMANN
}

\author{
ILDUS NURGALIEV \\ Department of Physics, Moscow State Timiryazev University, Timiryazevskaya Str., 49 \\ Moscow, 127550, Russia \\ ildus58@mail.ru \\ Received 15 June 2011 \\ Revised 25 June 2011
}

\begin{abstract}
It is shown that taking into consideration vorticity-related component of cosmological motion averts singularity and explains acceleration of the expansion. No mystic matter with dark energy is needed. That energy belongs to the local rotational motion (vorticity). Einstein's idea on static universe occurs to be encompassing conception for the standard cosmology, as well as for the emerging new (oscillating) one. Alexander Friedman gave to us its verbal model and was the most well prepared to give mathematical one.
\end{abstract}

Keywords: Cosmology; singularity; bounce; vortex; dark energy.

PACS numbers: 98.80.-k

\section{Introduction}

The year 1917 can be considered as the year of revolution in physics because equations of the theory applicable to all Universe were solved first time in the history of mankind. Friedmann from Russian Petrograd and abbey Lemaitre from Belgium were the first who did this.

An old question in theoretical cosmology was whether an infinitely static or oscillatory universe which avoids an initial singularity can be consistently constructed. Traditional answer is negative. As it was realized by Friedmann ${ }^{1}$ and especially by Tolman ${ }^{2,3}$ the fundamental obstacle is the second law of thermodynamics which dictates that the entropy increases from cycle to cycle. In the middle of the 20th century Hawking and Penrose theorems were accepted as final argument that classical general relativity necessarily has cosmological singularities.

Einsten's Universe was static not only because of mathematical simplicity but it was metaphysically, religiously and aesthetically predetermined in European way of thinking. Well known revelation on the "biggest blunder" seems to be blunder itself rather than cosmological term was a week idea. On the opposite, introducing cosmologic term instead of ignoring in the frame of Riemannian geometry second curvature (torsion) was a natural attempt. We are witnessing last decades how 
cosmological observations demand the factor for the measurements, results of which were interpreted as accelerated expansion, just like the factor (cosmological term) was needed for compensating regular gravitational cosmological contraction in 1917 . The role that cosmological torsion field in the frame of Einstein-Cartan theory acting as the cosmologic term was shown in Ref. 4. Exact cosmologic solution of the Einstein universe type and its analysis in terms of sustainability and linear perturbation stability were presented in Ref. 5. The conceptual re-incarnation of the Einstein universe presented in this report is based on taking into consideration vortexual component of the vector fields.

\section{New Law of Cosmologic Expansion}

This statement, expected by author to be met by many as a heretic one, means that the cosmologic singularity is a consequence of using simplistic expansion law instead of the realistic

$$
V_{\alpha}=H_{\alpha \beta} R^{\beta} .
$$

$H_{\alpha \beta}$ is distortion tensor (affinor), $R^{\beta}$ is radius-vector of galaxies. Excessive symmetric character of the Hubble's law is nothing but oversimplification of the cosmological principles of homogeneity and isotropy. The law given above includes distributed rotation which is imperative attribute of cosmological kinematics and has been ignored in the Hubble's law. (Was not the intuitive feeling of the oversimplification of the expansion law the reason for the contradictory attitude to expansion interpretation of redshift-distance correlation by Hubble himself?)

Historically earliest cosmological models with rotation are known as Godel solution of the Einstein equations Ref. 6. The development of this models and review of the models based on Godel type rotation where given, for example, in Ref. 710. It is important to note that in contrast to the local rotation presented in this report Godel type models are based on the global rotation. Therefore they are not isotropic. The given model has all the symmetries of the standard model because of the presence of the nonzero only vorticity square, but vorticity linear is averaged out as well as peculiar velocities.

Historically, looking a few centuries back, lack of given tensor (affinor) law might be considered the reason for the idea of the Cartesian vortexes to be less popular in comparison to the ideas of Newtonian potential forces. But if to look a few thousands years back, emerging cosmological model was and still is in harmony with oldest world religions. But new world religions standing on the "beginning" conception (creation), supported "singularizm" and won tender for cooperation with Science on the European scene.

The centrifugal forces acting between particles rotating randomly around each other are shown below to be able to reverse gravitational collapse. It is shown in this report that contribution from vortex $\omega$ (anti-symmetric part of $H_{\alpha \beta}$ ) provides stabilization of cosmological collapse and induces rotational structure formation. 
The dynamic equations are tensoral

$$
\dot{H}_{i j}+H_{i k} H_{k j}=-F_{i j} .
$$

It is natural to interpret increasing redshift for larger distances as the transverse red shift effect. The exact expression and one for the small $R H / c$ and $R \omega / c$, respectively, hold

$$
\frac{\nu}{\nu_{0}}=\frac{\left[1-\frac{R^{2}\left(\omega^{2}-H^{2}\right)}{c^{2}}\right]^{1 / 2}}{1-\frac{R H}{c}}, \quad \frac{\nu}{\nu_{0}}=1-\frac{R H}{c}+\frac{3}{2}\left(\frac{R H}{c}\right)^{2}-\frac{1}{2}\left(\frac{R \omega}{c}\right)^{2},
$$

where, according to cosmological principle, we put

$$
H_{i j}=\left(\begin{array}{ccc}
H & \pm \omega & \pm \omega \\
\mp \omega & H & \pm \omega \\
\mp \omega & \mp \omega & H
\end{array}\right) .
$$

Therefore, the most natural candidate for acceleration cause is "local rotation" $\omega>3 H$ square of which is homogenous and isotropic $\langle\omega\rangle=0,\left\langle\omega^{2}\right\rangle \neq 0$. The same is valid for the symmetrical traceless component (shear). Note, that (2) has generally all 9 components nontrivial therefore the obvious question about the sources of the "nontraditional" dynamic components will be published soon (firsthand in arxiv).

\section{Cosmological Expansion Started from the Big Bounce upon Local Rotation}

Local rotations (vortexes) play the radical stabilization role against cosmological singularity via the negative nonlinear contribution in energy in the retrospective extrapolation and making static or steady-in-the-average state of the universe (or a local region) possible. Therefore Einstein could "permit" the galaxies to rotate instead of postulating lambda-term ad hoc in the case of general relativistic consideration of static in timely averaged Universe Ref. 1. Though, as we know, it dose not mean necessarily that the lambda-term is not needed on the bases of other arguments. The further mathematical considerations below are done in the frame of the Newtonian formalism shifting from Riemann-Cartan geometry because of the clear purpose: to emphasize that phantom of cosmological singularity is emerged neither because of general relativity (Riemannian character of the space-time), nor because of special relativity (limitedness of the physical velocities), but because of ignoring vorticity, excessive symmetry hypothesis. Therefore, averting cosmological singularity happens not necessarily because of involving Cartan torsion but because of returning the rights to vorticity to be counted. To understand this statement it is enough to know Newtonian hydrodynamics in Euclidean geometry regardless the conclusions of advanced geometries.

Let us consider local imaginary spherical region of the homogeneous and isotropic infinite distribution of gravitating "dust". As Milne and McCrea did, we 
can ignore the surrounding matter (Birkhoff theorem). But in contrary to Milne and McCrea Ref. 11, we do not demand the test particle rest at the contracting sphere marking the boundary of the ball of the constant mass but let it move with the typical peculiar cosmological velocity $\vec{v}_{\text {pecular }}$ on the sphere because rotation is a typical motion in the Universe along with well measured expansion, and the galaxies do have peculiar components of their motion. In other words we rehabilitate vorticity, and thereby long time ignored centrifugal cosmological forces as well. $\vec{v}_{\text {pecular }}$ is perpendicular to pure Hubble expansion. It is ignored component of the cosmological motion in the standard general relativistic Friedmann-Lemaitre models ${ }^{3}$ as well. In Newtonian cosmology we derive in traditional notations:

$$
\dot{H}+H^{2}=K^{2} \rho^{4 / 3}-(4 \pi G / 3) \rho .
$$

Here we have got good surprise. The same functional dependence of $\omega^{2}$ on $R$ as of energy density and pressure of ultra-relativistic matter (electromagnetic radiation, photons gas), all of them while isotropic, are proportional to $1 / R^{4}$, and the very same law of conservation of the averaged shear squared $\sigma^{2}$ (the latter causes black "matter" effect along with "black energy" effect of $\left.\omega^{2}\right)$ remain the functional character of (2) unchanged causing only the re-defining the constant $K^{2}=\Omega^{2}-\Gamma^{2}-\Sigma^{2}$, where constants $\Omega, \Gamma, \Sigma$ stand for vortex, radiation (energy density and pressure) and shear constants in corresponding conservation laws. It is important to note that the dynamic system (3) is isomorphic to general relativistic cosmologic equation because radiation term and vortexial term get formed single $\rho^{4 / 3}$-term.

Here is the first integral of (2): $H^{2} / 2=-K^{2} / 2 R^{4}+G M / R^{3}+A / R^{2}$, where $A$ is a constant of integration. Let us write down the final integral of the cosmologic equation. For $A \geq 0$ we have

$$
\begin{aligned}
t+t_{0}= & -(2 A)^{-1}\left(2 A R^{2} K+2 G M R-K^{2}\right)^{1 / 2}-G M(2 A)^{3 / 2} \ln \left(2 ^ { 3 / 2 } A ^ { 1 / 2 } \left(2 A R^{2}\right.\right. \\
& \left.\left.+2 M G R-K^{2}\right)+4 A R+2 G M\right) .
\end{aligned}
$$

For $A \leq 0$ we have

$$
\begin{aligned}
t+t_{0}= & (2 A)^{-1}\left(2 A R^{2} K+2 G M R-K^{2}\right)^{1 / 2}-G M(-2 A)^{3 / 2} \arcsin [(2 A R+G M) \\
& \left./\left(2 A^{2} K^{2}+G M^{2}\right)^{1 / 2}\right] .
\end{aligned}
$$

What is exact value of $\omega^{2}$ in the radiation-dominated Einstein static Universe? We have $\omega^{2}=(4 \pi G / 3) \rho_{\text {radiation }}=\left(4 \pi G / 3 c^{2}\right) a T^{4}$, where $a=\pi^{2} k^{4} / 15 \hbar^{3} c^{3}=7.56 \times$ $10^{-16} \mathrm{~J} / \mathrm{m}^{3} \mathrm{~K}^{4}$. Therefore, $\omega^{2}=\left(4 \pi^{3} G k^{4} / 45 \hbar^{3} c^{5}\right) T^{4}$. Or, compactly, $\omega^{2}=a \chi T^{4}$, and $\omega^{2}=\varkappa T^{4}$, where $\chi=4 \pi G / 3 c^{2}=3.1 \times 10^{-27} \mathrm{~m} / \mathrm{kg} . \varkappa=4 \pi^{3} G k^{4} / 45 \hbar^{3} c^{5}=$ $2.11 \times 10^{-41} \mathrm{~s}^{-2} \mathrm{~K}^{-4}$ is a new universal constants combination. The illegally trampled right to rotate is returned to point of matter (particle). For $T=2.7 \mathrm{~K}$ we get that even such a small value as $\omega^{2} \approx 1.12 \times 10^{-40} \mathrm{rad}^{2} / \mathrm{s}^{2}$ could be enough to compensate the radiation contribution to the cosmological contraction preceded to the observed expansion, i.e. less than, supposedly, existing value. For static Einstein universe if according WMAP data density of dark energy is $9.9 \times 10^{-30} \mathrm{~g} / \mathrm{cm}^{3}$, then $\omega \approx 9.15 \times 10^{-20} \mathrm{~s}^{-1}$. 


\section{Conclusions}

We have given an answer "no" to the following hypothesis existed in the standard cosmology based on Hubble expansion which almost steadily was rejected by Hubble. In the Universe, somebody with some unknown purpose, at some mysterious previous stage of its evolution had fine tuned with 100\%-precision the zero scattering of each particle around each other (Hubble law). This entity provided by this fantastic job the delivery all of them to the very same point at the very same time. This, however, does not explain ${ }^{12-14}$ our Universe. The point is that galaxies rotate. Rotation is common for any object even for a "material point" which is a small material body. Alexander Friedman gave us verbal model of the cyclic cosmology and was the most well prepared to give mathematical embodiment for this realistic model very soon after his famous solutions being highly qualified theoretician in Meteorology. We come to the brand new conception which author suggests to call "Zillions of bounces" conception instead of the single Big Bang conception.

So the line of Spinoza and Einstein (eternal Universe) might be continued soon. But, unfortunately, Alexander Friedmann passed away even sooner, in his very young age. And creationistic "singularizm" gained its temporary (nearly one century long so far) victory. The end of the "singularizm" period deals with the fact that not only first curvature but the second one as well (torsion, mentioned above) have to be accounted for including the fact that small element of matter (material point) can rotate.

\section{References}

1. A. Friedmann, Z. Phys. 10, 377 (1922).

2. R. C. Tolman, Phys. Rev. 38, 1758 (1931).

3. R. C. Tolman, Relativity, Thermodynamics and Cosmology (Oxford University Press, Oxford, 1934).

4. I. S. Nurgaliev and V. N. Ponomarev, Russ. Phys. J. 25, 929 (1982).

5. I. S. Nurgaliev and V. N. Ponomarev, Phys. Lett. B 130, 378 (1983).

6. K. Godel, Rev. Mod. Phys. 21, 447 (1949).

7. D. D. Ivanenko, V. G. Krechet and V. F. Panov, Rotation of the Universe and cosmology, in Gravitation and General Relativity, ed. V. R. Kaygorodov (Kazan University Press, Kazan, 1987), p. 33.

8. V. F. Panov, Russ. Phys. J. 28, 18 (1985).

9. V. G. Krechet and V. F. Panov, Astrophysics 28, 400 (1988).

10. V. G. Krechet, Grav. Cosm. 10, 153 (2004).

11. W. A. McCrea and E. A. Milne, Q. J. Math. (Oxford) 5, 73 (1934).

12. I. S. Nurgaliev, Singularities are averted by vortex, in 13th Gravitational Conference - International Conference on Gravitation, Cosmology and Astrophysics, ed. A. P. Efremov (PFUR, Moscow, 2008), p. 117.

13. I. S. Nurgaliev, Nonsingular Universe, in Modern Problems of Gravitation, Cosmology and Relativistic Astrophysics, ed. V. N. Melnikov (PFUR, Moscow, 2010), p. 92.

14. I. S. Nurgaliev, Grav. Cosm. 16, 313 (2010). 